\title{
OC48 - Hurtology: an online course
}

\author{
Casey Frechette (US) ${ }^{1}$; Barbara Frechette ${ }^{2}$ \\ ${ }^{1}$ USF St. Petersburg, Florida; ${ }^{2}$ Formerly at Shenandoah University
}

Theme: School health.

Keywords: Computer game, hurt, mental health, young people.

Background: The prevalence of anxiety and depression suggests a need to improve on the mental health education of young people. The school setting can provide a venue for offering such knowledge to adolescents.

Objectives: This study explored whether a video game enhances lessons designed to help adolescents become more receptive to learning about mental health concepts.

Methods: This study used a quantitative between-subjects design. The first group experienced a set of computer-based lessons. The second group received the same content, but also played a video game designed to reinforce topics explored in the other materials.

Results: The findings showed that game players demonstrated deeper learning on at least one measure.

Discussion: Helping adolescents develop better ways to understand the relevance of emotional health is a worthwhile endeavour.

Conclusions: New technologies can be used to improve learning and help young people become more receptive to addressing mental health concerns. 